\title{
Correction of Phase Effects Produced by Eddy Currents in Solvent Suppressed ${ }^{1} \mathrm{H}$-CSI
}

\author{
Joseph R. Roebuck, David O. Hearshen, Matthew O’Donnell, Tom Raidy
}

\begin{abstract}
Accurate phasing of MRS spectra is often difficult unless time varying phase effects produced by gradient-induced eddy currents that persist during data acquisition are eliminated. This effect is particularly problematic in ${ }^{1} \mathrm{H}-\mathrm{CSI}$ spectra where frequency shifts produced by static fleld inhomogeneity and phase shifts produced by eddy currents combine. In this paper we present a method that corrects both shifts and eliminates manual phasing of individual CSI spectra typically required to recover a pure absorption line shape. The method uses a time domain phase correction derived from the ambient water signal acquired under identical conditions (i.e., acquisition parameters, gradient sequence) as the solvent-suppressed CSI data. Results from CSI experiments on phantoms and in vivo solvent suppressed $\mathrm{H}$-CSI spectra from normal human brain are presented demonstrating the capabilities of the technique.
\end{abstract}

Key words: CSI; eddy-currents; phase correction; phantom.

\section{INTRODUCTION}

Proton chemical shift imaging ( $\left.{ }^{1} \mathrm{H}-\mathrm{CSI}\right)$ has become an increasingly popular technique for performing spatially localized in vivo MRS. This is particularly true in the human brain, where a number of biochemically important species are observable and spectral contamination from fat can be minimized (1-8). The primary advantage of CSI is that localized spectra from multiple locations can be simultaneously acquired $(9,10)$. In this respect, it is significantly more time efficient than single voxel techniques, however, it is relatively more difficult to implement. The primary difficulty with ${ }^{1} \mathrm{H}-\mathrm{CSI}$ is the large influence of static field inhomogeneity and gradient-induced eddy currents. These two factors can distort the resonance frequency, line width, and line shape (i.e., overall phase) effectively compromising these data and necessitating significant postprocessing for proper spectral interpretation.

Several techniques have been suggested for correcting the effects of static field inhomogeneity on CSI data (1113), but these techniques do not consider the additional

\section{MRM 30:277-282 (1993)}

From the Bioengineering Program, University of Michigan, Ann Arbor, Michigan (J.R.R., M.O.); the Department of Diagnostic Radiology, Henry Ford Hospital, Detroit, Michigan (J.R.R., D.O.H.); the Department of Electrical Engineering and Computer Science, University of Michigan, Ann Arbor, Michigan (M.O.); and General Electric Medical Systems, Milwaukee, Wisconsin (T.R.)

Address correspondence to: Joseph R. Roebuck, B.S. M.S., Division of MR (WCB), Henry Ford Hospital, 2799 West Grand Boulevard, Detroit, MI 48202.

Fieceived December 28, 1992; revised May 17, 1993; accepted May 18, 1993.

This work was originally presented at the 11th Annual Meeting of the Society of Magnetic Resonance in Medicine, Berlin, Germany.

0740-3194/93 $\$ 3.00$

Copyright (C) 1993 by Williams \& Wilkins

A.ll rights of reproduction in any form reserved. time varying phase effects produced by eddy currents that persist during data acquisition. These phase effects can lead to improper spectral interpretation, especially if compounded by incomplete solvent suppression, overlapping resonances, and resonance frequency shifts. A phase correction that addresses this problem was developed using nonlocalized spectra $(14,15)$ and extended to single voxel STEAM spectra $(16,17)$. These corrections use an unsuppressed signal from ambient water acquired under identical conditions as the solvent-suppressed signal to calculate the eddy current contribution to the phase of the solvent-suppressed signal. This contribution is then eliminated leaving only the true chemical shift component. In this paper, we extend the eddy current correction to ${ }^{1} \mathrm{H}$-CSI data and consider in detail the effects of using a number of strategies for time-dependent phase correction.

\section{METHODS}

The signal acquired in a spatially localized MRS experiment can be modeled as

$$
s(t)=\sum_{n=1}^{N} \int M_{n}\left(\mathbf{r}^{\prime}\right) \exp \left\{-t / T_{2_{n}}\right\} \exp \left\{i \phi\left(\mathbf{r}^{\prime}, \sigma_{n}, t\right)\right\} d \mathbf{r}^{\prime},
$$

where the integration is taken over a voxel centered at $\mathbf{r}=(x, y, z)$ with dimensions $(\Delta x, \Delta y, \Delta z)$ and $M_{n}(\mathbf{r})$ is the transverse magnetization of the $n^{\text {th }}$ spin species having shielding constant $\sigma_{n}$ and spin-spin relaxation time $T_{2_{n}}$. The summation is taken over all $N$ spin species contributing to the signal. The phase term in Eq. [1] is given by

$\phi\left(\mathbf{r}^{\prime}, \sigma_{n}, t\right)=\int_{0}^{t} \gamma\left[B_{0}+\Delta B_{0}\left(\mathbf{r}^{\prime}, t^{\prime}\right)\right] \cdot\left[1-\sigma_{n}\right] d t^{\prime}+\phi_{o}$,

where the integration starts at the time phase coherence is established (nominally the end of the last RF pulse). $B_{0}$ is the static magnetic field strength, $\Delta B_{0}(\mathbf{r}, t)$ is the magnetic field variation produced by static field inhomogeneity and eddy currents, and $\phi_{\mathrm{o}}$ is the offset between the rotating frame and the absorption mode detector phase. In practice, $s(t)$ is acquired using phase-sensitive detection and can be expressed in complex notation as

$$
s(t)=|s(t)| \exp \{i \Phi(t)\}=s_{r}(t)+i \cdot s_{i}(t),
$$

where $|s(t)|$ is the magnitude, $\Phi(t)$ is the aggregate phase, and $s_{r}(t)$ and $s_{i}(t)$ are the real and imaginary components of $s(t)$, respectively.

Equations [1] and [2] show how static field inhomogeneity and eddy currents affect the phase of $s(t)$. If $\left|\Delta B_{0}\right|$ 
varies with position, a distribution of isochromats is produced across the voxel and $s(t)$ is derived from the vector sum of phasors arising from each isochromat weighted by the corresponding values of $M_{n}$. If $\left|\Delta B_{0}\right|$ varies with time, the cumulative phase of each isochromat, and hence $\Phi(t)$, is a function of $\Delta B_{0}$ as well as chemical shift. The phase term in Eq. [1] cannot be removed from the integral unless $\left|\Delta B_{0}\right|$ is spatially invariant. If we split the integral in Eq. [2], then the $B_{0}$ and $\phi_{o}$ components of the phase can be removed from the integral in Eq. [1]. Furthermore, if we assume that $1 \gg \sigma_{n}$, the chemical shift component of the phase can also be removed from the integral in Eq. [1].

Because phase effects produced by $\Delta B_{\mathrm{o}}(\mathbf{r}, t)$ are also a function of the gyromagnetic ratio $(\gamma)$, they are most pronounced in ${ }^{1} \mathrm{H}$-MRS. Coincidentally, the gradient requirements for ${ }^{1} \mathrm{H}$-MRS are significant due to solvent suppression and localizing relatively small voxels. Phase effects can be greatly reduced in a solvent-suppressed ${ }^{1} \mathrm{H}$-MRS signal by performing a differential measurement of the solvent suppressed (or sample) and unsuppressed (or reference) signals. If we acquire sample and reference signals from the same voxel under identical conditions (i.e., acquisition parameters, gradient sequence), the spatial and temporal variation of $\Delta B_{0}$ across the voxel remains constant between acquisitions. If we also assume that the magnetic field does not vary significantly across the voxel, the $\Delta B_{0}$ component of the phase can be removed from the integral in Eq. [1] and the aggregate phase of the two signals are identical with one another except for phase contributions due to chemical shift. Finally, if we disregard the phase contributions of nonsolvent spins in the reference and keep the carrier frequency and phase offset constant between acquisitions (a practical requirement in CSI), the sample and reference signals are given by

$$
S(t)=|S(t)| \exp \left\{i\left(\phi_{\delta}(t)+\phi_{\omega}(t)+\phi_{e}(t)+\phi_{o}\right)\right\},
$$

and

$$
R(t)=|R(t)| \exp \left\{i\left(\phi_{\omega}(t)+\phi_{e}(t)+\phi_{o}\right)\right\},
$$

respectively. The phase contributions due to chemical shift, resonance frequency offset, eddy currents, and phase offset are denoted $\phi_{\delta}(t), \phi_{\omega}(t), \phi_{e}(t)$, and $\phi_{o}$.

The $\phi_{\omega}(t), \phi_{e}(t)$, and $\phi_{o}$ components in Eq. [4] can be eliminated by multiplying $S(t)$ by a correction function derived entirely from $R(t)$ and given by

$$
\begin{aligned}
C(t) & =\exp \left\{-i\left(\phi_{\omega}(t)+\phi_{e}(t)+\phi_{o}\right)\right\}=\frac{R^{\star}(t)}{|R(t)|} \\
& =\frac{1}{|R(t)|}\left\{R_{r}(t)-i \cdot R_{i}(t)\right\} .
\end{aligned}
$$

The corrected sample signal, $\hat{S}(t)=S(t) \cdot C(t)$, retains chemical shift information, is referenced to the Larmor frequency $\gamma B_{0}$, is free of phase distortions due to eddy currents, and is in phase with the detector (i.e., $\hat{S}(\omega)=$ $\mathscr{F}\{\hat{S}(t)\}$ has a pure absorption line shape).

In a ${ }^{1} \mathrm{H}$-CSI experiment, multiple spatially localized signals are generated from voxels spanning the field of view, where the $k^{\text {th }}$ signal, $s_{k}(t)$, arises from the voxel centered at $\mathbf{r}=\left(x_{k}, y_{k}, z_{k}\right)$ with dimensions $\left(\Delta x^{\prime}, \Delta y^{\prime}, \Delta z^{\prime}\right)$. In this case, each CSI voxel is subjected to a different magnetic field distribution depending on the spatial dependence of $\Delta B_{0}(\mathbf{r}, t)$. If the contribution of static field inhomogeneity to $\Delta B_{0}(r, t)$ is significant, the distribution of isochromats in the sample will vary between voxels producing differences in line width and resonance frequency between the $s_{k}(t)$. If the spatial contribution of the eddy currents to $\Delta B_{0}(\mathbf{r}, t)$ is significant, the cumulative phase of each isochromat will also vary between voxels producing differences in the overall phase between the $s_{k}(t)$. In both cases, the phase terms in Eq. [4] and [5] are spatially dependent. This spatial dependence can produce significant differential phase effects between individual CSI voxels that must be considered when phase correcting CSI spectra.

The phase correction described above can be applied to ${ }^{1} \mathrm{H}$-CSI data sets, providing sample and reference signals are acquired under identical conditions. This eliminates phase contributions due to frequency offsets and eddy currents by accounting for the differential effect of $\Delta B_{0}(\mathbf{r}, t)$ on individual voxels. Time domain signals are first Fourier transformed in the phase encoding direction, eliminating the phase factors associated with encoding spatial information, but not the phase offset $\phi_{o}$. If the spatially localized signals in the sample and reference CSI data sets are denoted $S_{k}(t)$ and $R_{k}(t)$, the corresponding correction functions $\left(C_{k}(t)\right)$ are evaluated using the respective $R_{k}(t)$ in Eq. [6] and the corrected sample signals are given by $\hat{S}_{k}(t)=S_{k}(t) \cdot C_{k}(t)$. Note that differences in magnetic field inhomogeneity leading to resonance frequency shifts between voxels are eliminated and each $\hat{S}_{k}(t)$ is referenced to the Larmor frequency. Furthermore, if $\phi_{o}$ is constant between acquisitions, each $\hat{S}_{k}(t)$ is in phase with the detector.

Acquiring sample and reference data under identical conditions can exact a significant time penalty, particularly in multidimensional CSI. Differences in signal-tonoise ratio (SNR) between sample and reference notwithstanding, additional time is required for phase encoding and phase cycling in the reference that might otherwise be spent elsewhere. Reducing the reference acquisition time by using slightly different acquisition parameters is an alternative; however, this may not suffice for adequate correction. In the figures that follow, a sample CSI correction is performed using a reference acquired under identical conditions (i.e., number of phase encodes, spatial resolution, acquisition parameters, gradients). This correction is compared with a number of compromise strategies that reduce reference acquisition time.

\section{RESULTS}

${ }^{1} \mathrm{H}$-MRS data were acquired at $1.5 \mathrm{~T}$ using a whole-body MR system and standard birdcage head coil driven in quadrature (GE Medical Systems, Milwaukee, WI). The phantom consisted of two concentric glass spheres $(\mathrm{D} \approx$ $5,20 \mathrm{~cm}$ ) as illustrated in Fig. 1. The inner and outer spheres contained $50 \mathrm{mM}$ lactate (Lac) and $50 \mathrm{mM}$ creatine $(\mathrm{Cr})$, respectively. The phantom was positioned near 


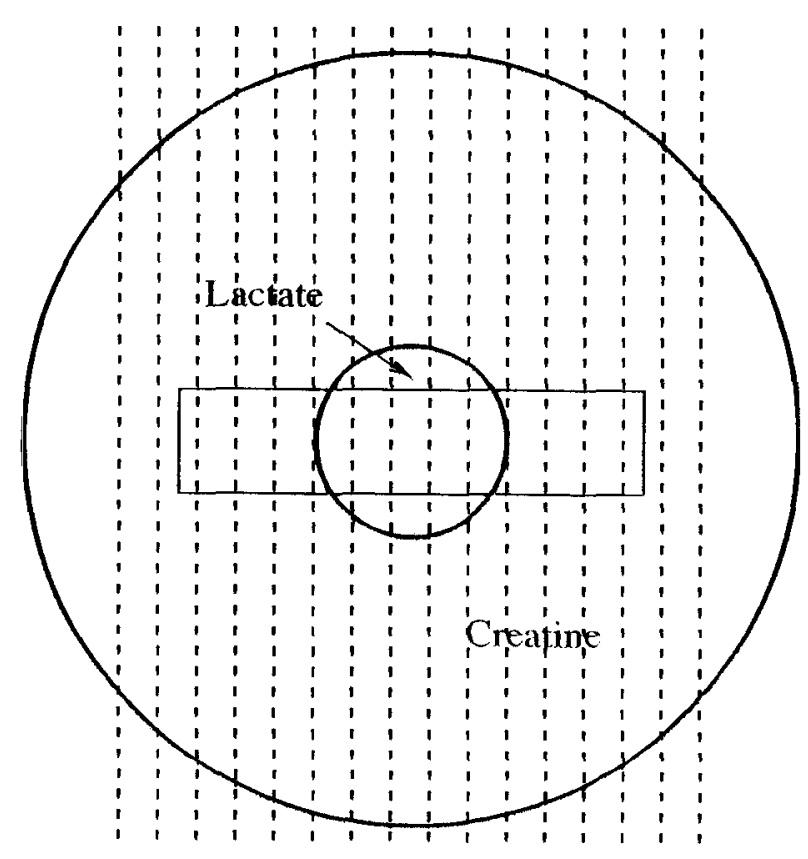

$F \| G$. 1. Experimental setup. The inner and outer compartments of the phantom contained $50 \mathrm{mM}$ Lac and $50 \mathrm{mM} \mathrm{Cr}$, respectively. A STEAM voxel was defined across the inner compartment, and $1 D$-CSI data were acquired using phase encoding along the long axis of the STEAM voxel.

isocenter where a $2 \times 2 \times 12 \mathrm{~cm}$ voxel was defined across the inner compartment using STEAM (18). Static field homogeneity was optimized across the voxel using first order shimming (19) and solvent suppression was performed using a three CHESS pulse strategy (20) in conjunction with Shinnar-Le Roux pulses (21). One-dimensional phase encoding (PE) was performed along the long axis of the voxel and produced $122 \times 2 \times 1 \mathrm{~cm}$ subvoxels in the 1D-CSI data set. Sample CSI data were acquired using $16 \mathrm{PE}$ steps with eight phase cycles/PE and a 2000-ms repetition time (TR), a 20-ms echo time (TE), and an 11-ms mixing time (TM). Reference CSI data were acquired using $16 \mathrm{PE}$ steps with various TRs and number of phase cycling steps; however, all other acquisition parameters were held constant between the sample and reference experiments. In particular, crusher gradients associated with solvent suppression were still used during reference acquisition. Single voxel reference data from the entire STEAM voxel were also acquired using the same acquisition parameters as the sample CSI (i.e., eight phase cycles, a 2000-ms $T R$, a 20-ms $T E$, and an 11-ms TM). In all cases, 512 time domain samples were captured at a sampling rate of $1 \mathrm{KHz}(2 \mathrm{~Hz}$ frequency resolution).

Data processing was performed on a SUN SparcStation using the SA/GE software package (GE Medical Systems) in conjunction with user developed programs. Data processing required no operator interaction and took approximately $2 \mathrm{~min}$ to complete. Several corrections were compared using the same sample CSI data set. In each case, the sample CSI was Fourier transformed in the PE direction prior to phase correcting each $S_{k}(t)$. Phase cor- rection was either performed manually (Fig. 2) or by multiplying each $S_{k}(t)$, point-for-point, by the corresponding correction function (Figs. 3-8). Manual phase correction consisted of adjusting the zero-order phase ( $\phi_{o}$ in Eq. [2]) of one spectrum in the CSI data set to obtain a flat baseline and lineshapes approximating absorption mode spectra; this phase correction was then applied to all the CSI spectra. Note that no higher order manual phasing was performed. For those corrections involving reference CSI data, the reference CSI was Fourier transformed in the PE direction prior to calculating the corresponding correction function associated with each $R_{k}(t)$. For those corrections involving single voxel reference data, each $S_{k}(t)$ was corrected using the same single voxel reference signal. Following phase correction, localized sample signals were zero padded to 1024 points and Fourier transformed to produce localized chemical shift spectra. Unless otherwise noted, no additional phasing, shifting, apodization, or interpolation was performed. All phantom spectra are referenced to water at $4.75 \mathrm{ppm}$.

Figure 2 shows the effects of eddy currents on CSI data. Lac from the inner compartment is present in the center five spectra and $\mathrm{Cr}$ from the outer compartment is present in the remaining seven spectra. Each signal in the sample data was manually phased using the phase offset estimated (by visual inspection) for the center spectrum. Eddy current induced distortions in these spectra are seen as negative excursions on both sides of isocenter in $\mathrm{Cr}$ and in Lac near isocenter. In addition, Lac has an asymmetric line shape. Figure 3 shows the correction with a reference acquired under identical conditions as the sample (i.e., $16 \mathrm{PE}$, eight phase cycles/PE, a 2000-ms $T R$, a $20-\mathrm{ms} T E$, and an 11-ms TM). The negative excursions seen in Fig. 2 have been eliminated and Lac has a symmetric line shape.

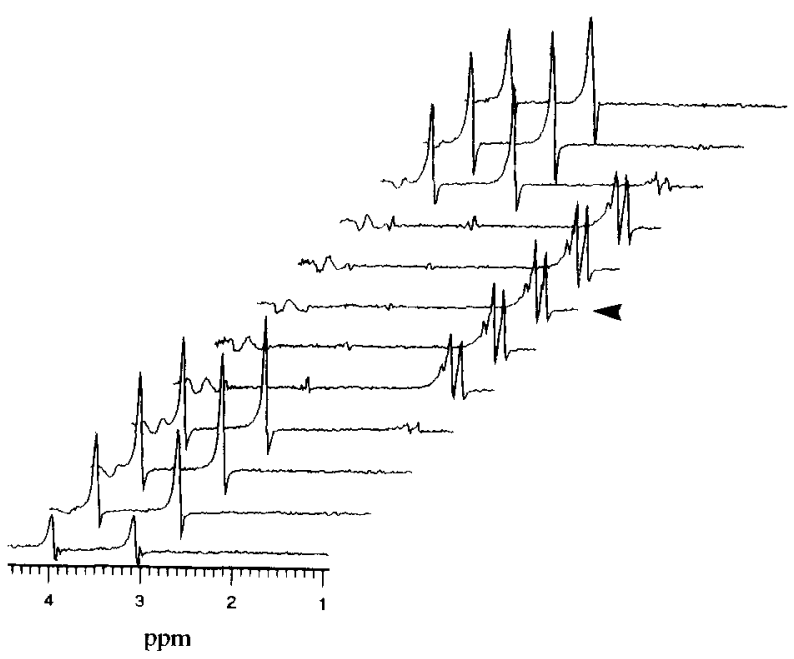

FIG. 2. Manually phased sample CSI. Lac is present in the center 5 spectra and $\mathrm{Cr}$ in the remaining 7 spectra. Eddy current induced distortions are seen as negative excursions in the $\mathrm{Lac}$ and $\mathrm{Cr}$ resonances and the asymmetric Lac line shape. The arrow indicates the spectrum selected for manual phasing. A zero-order phase correction of $3^{\circ}$ was applied to each spectrum. 


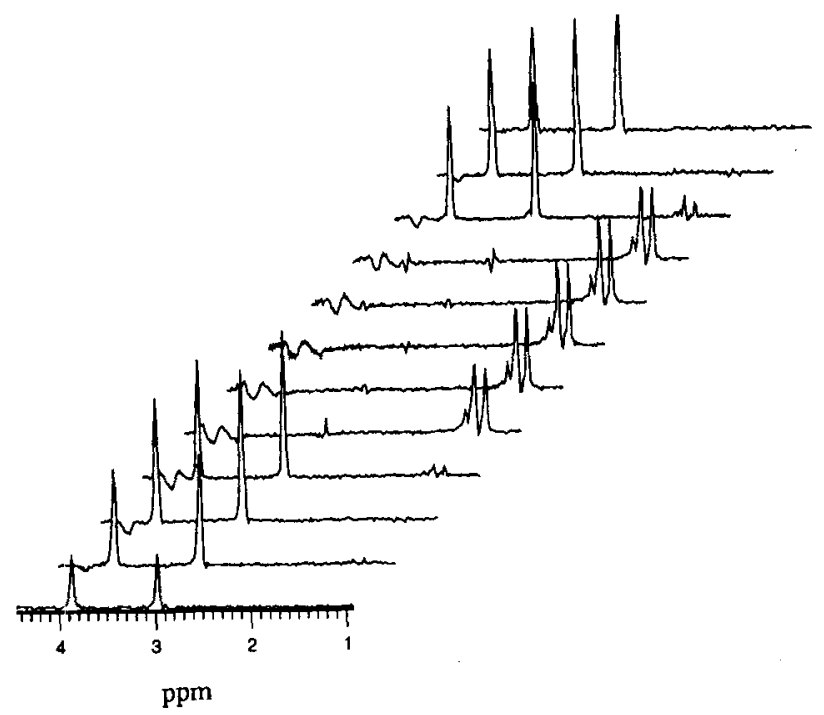

FIG. 3. Correction using identical reference CSI (16 PEs). Negative excursions have been removed in $\mathrm{Cr}$ and Lac and Lac has a symmetric line shape. Spectra show marked improvement compared with manually phased data (Fig. 2). The reference acquisition time was $272 \mathrm{~s}$.

Figures 4 to 7 show the corrections rendered with various compromise strategies intended to reduce the reference acquisition time. Figure 4 shows the correction rendered with fewer PEs from the reference. Reference data used here were derived from the middle eight PEs of the data described for Fig. 3. Zero padding to 16 points in the PE direction produced a spatially interpolated reference having the same number of PEs as the sample. The negative excursions have been largely removed in $\mathrm{Cr}$ to the left of isocenter (bottom spectra) and in the Lac spectra

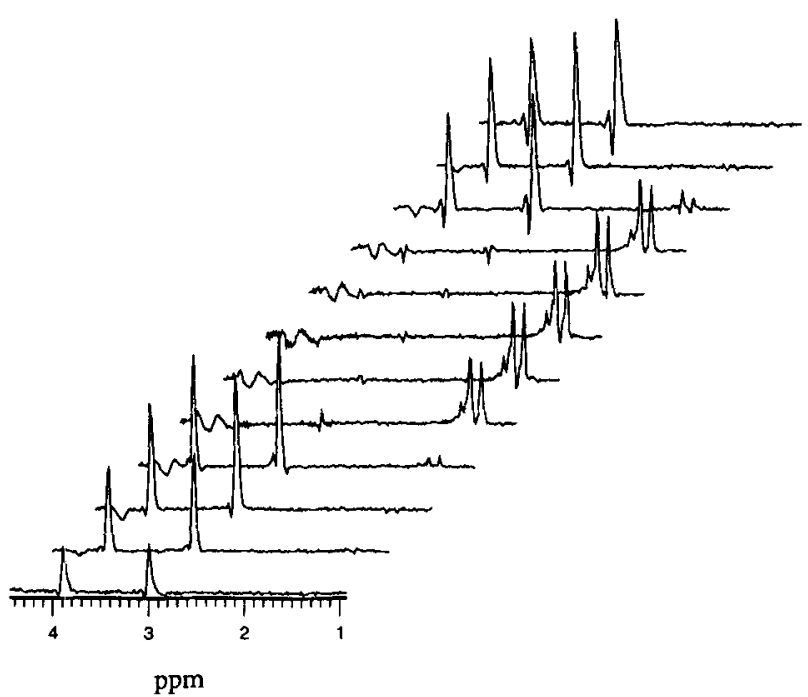

FIG. 4. Correction using fewer PEs in reference CSI (8 PEs). Negative excursions have been largely removed in Lac and the bottom $\mathrm{Cr}$ spectra and Lac has a symmetric line shape. Negative excursions remain in the top $\mathrm{Cr}$ spectra. Spectra are improved relative to manually phased data (Fig. 2) but are distorted compared with the identical reference CSI correction (Fig. 3). The middle 8 PEs can be acquired in $144 \mathrm{~s}$. and Lac has a symmetric line shape. Significant negative excursions remain, however, in $\mathrm{Cr}$ to the right of isocenter (top spectra). Figure 5 shows the correction rendered with a single voxel reference signal. Reference data used here was the signal acquired from the entire STEAM voxel with eight phase cycles and a $2000-\mathrm{ms} T R$, a $20-\mathrm{ms}$ $T E$, and an 11-ms TM. The negative excursions have been reduced in $\mathrm{Cr}$ (especially to the left of isocenter) and largely removed in the Lac spectra; however, asymmetries remain in the Lac line shape.

Figure 6 shows the correction rendered with fewer phase cycles and reduced reference TR. Reference data used here were acquired using $16 \mathrm{PE}$, two phase cycles/ PE, a 915-ms TR, a 20-ms TE, and an 11-ms TM. The negative excursions have been removed in both $\mathrm{Cr}$ and Lac, though there is somewhat less improvement in Cr to the right of isocenter (top spectra) as compared with a correction rendered by just reducing $T R$ (not shown). In addition, some asymmetry remains in the Lac line shape. Figure 7 shows the correction rendered with water referencing and a single voxel reference. Reference data used here were the single voxel signal described above. Prior to phase correction, the sample data were individually referenced to the single voxel reference data. This was accomplished by manually shifting the solvent resonance in each localized spectrum of the identical reference CSI to the solvent resonance frequency of the single voxel signal. Identical shifts were then applied to the corresponding spectra in the sample CSI. The frequency corrected sample was then inverse transformed and subjected to single voxel reference correction. The negative excursions in $\mathrm{Cr}$ and Lac appear largely unaffected by the single voxel correction.

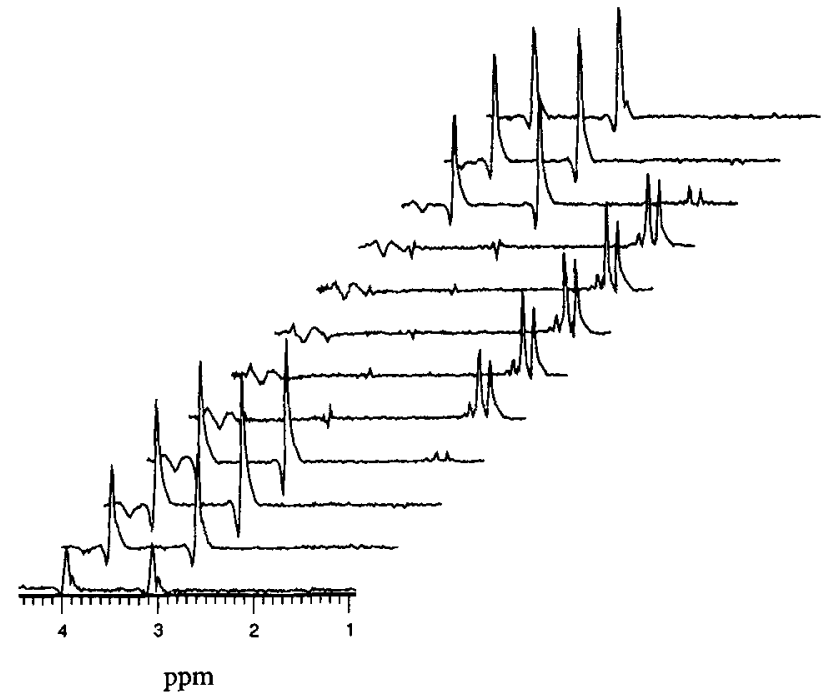

FIG. 5. Correction using single voxel reference. Negative excursions have been largely removed in the Lac spectra; asymmetries remain in the Lac line shape, however, as compared with the identical reference CSI correction (Fig. 3). Negative excursions have been reduced in the bottom $\mathrm{Cr}$ spectra compared with the manually phased data (Fig. 2). The reference acquisition time was $32 \mathrm{s.}$ 


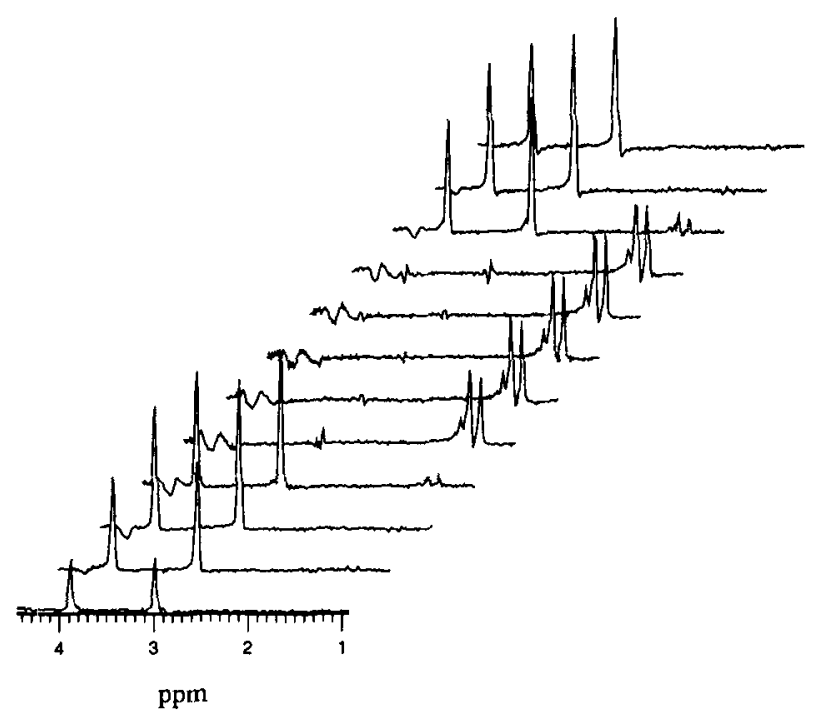

FIG. 6. Correction using fewer phase cycles and reduced TR reference CSI. Negative excursions have been removed in $\mathrm{Cr}$ and Lac, though there is somewhat less improvement in the top $\mathrm{Cr}$ spectra compared with just reducing $T R$ (no shown). The asymmetry in the Lac line shape is comparable with that obtained by just reducing $T R$. The reference acquisition time was $37 \mathrm{~s}$.

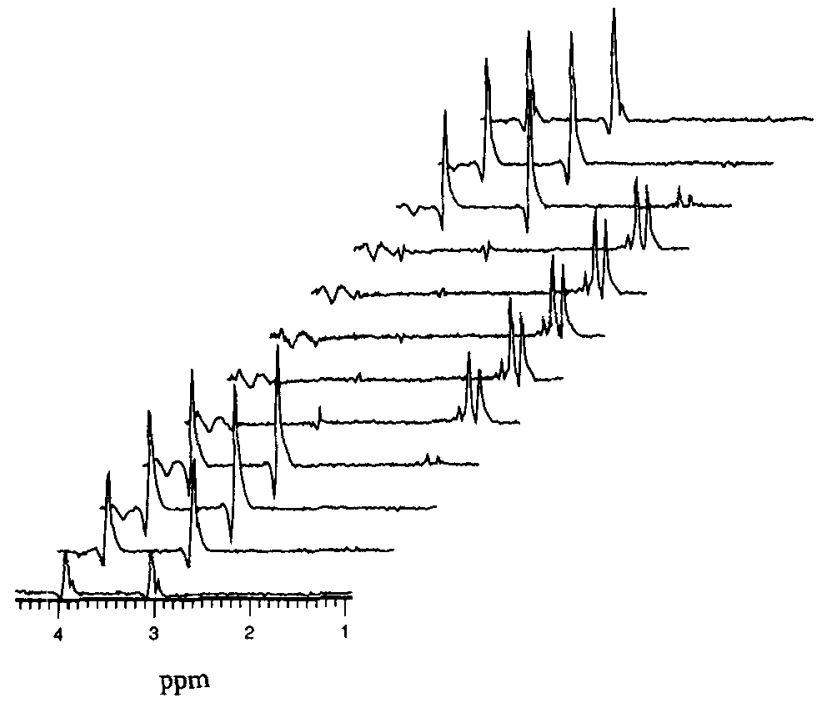

FIG. 7. Correction using water referencing and single voxel reference. Negative excursions in $\mathrm{Cr}$ and $\mathrm{Lac}$ are comparable with those seen in the single voxel correction (Fig. 2). Apart from expected frequency shifts, there are no other obvious effects on phase correction as compared with the single voxel correction.

\section{DISCUSSION}

The algorithm for automated phase correction was tested using in vitro data to objectively compare various time reduction strategies. The algorithm appears to be robust if used with a reference CSI acquired under identical conditions. In this case, the sample and reference CSI identically sample the spatial distribution of $\Delta B_{0}(\mathbf{r}, t)$. Furthermore, localized signals in the corrected sample were free of magnetic field inhomogeneity induced frequency shifts and eddy current induced line shape distortions and no additional phasing was required to obtain pure absorption line shapes.

Acquisition of an identical reference can significantly increase total exam time, particularly in multidimensional CSI. To reduce this additional time, various strategies were tested that approximate the phase effects produced by $\Delta B_{0}(\mathbf{r}, t)$ in the reference data. Reducing $T R$ and phase cycling in the reference CSI appears to have little effect on reducing the performance of the phase correction while providing a factor of eight in time savings. This implies that the eddy current time constants associated with this phantom and set of acquisition parameters are short compared with the TR of the experiment. Reducing the number of PEs in the reference CSI produced less favorable results. This implies that spatial interpolation of intermediate voxels in the reference does not adequately reproduce the spatial dependence of eddy currents in our phantom. Where the spatial dependence of the eddy currents is slowly varying (e.g., near isocenter and presumably to the left of isocenter), spatial interpolation appears to provide an adequate estimate of the reference phase for the correction. In the extreme, the correction using a single voxel reference signal appears to be inadequate, even when correcting the phase contribution due to local static field inhomogeneity using independent water referencing. This is because a single voxel correction cannot account for the spatial dependence of the eddy currents.

The algorithm described here is based on an assumption not generally valid in vivo. It assumes that the magnetic field does not vary significantly across the voxel that the sample and reference signals are acquired from. This approximation is reasonable in relatively small voxels located in regions where susceptibility effects are relatively small. As an example, Fig. 8 illustrates the effect

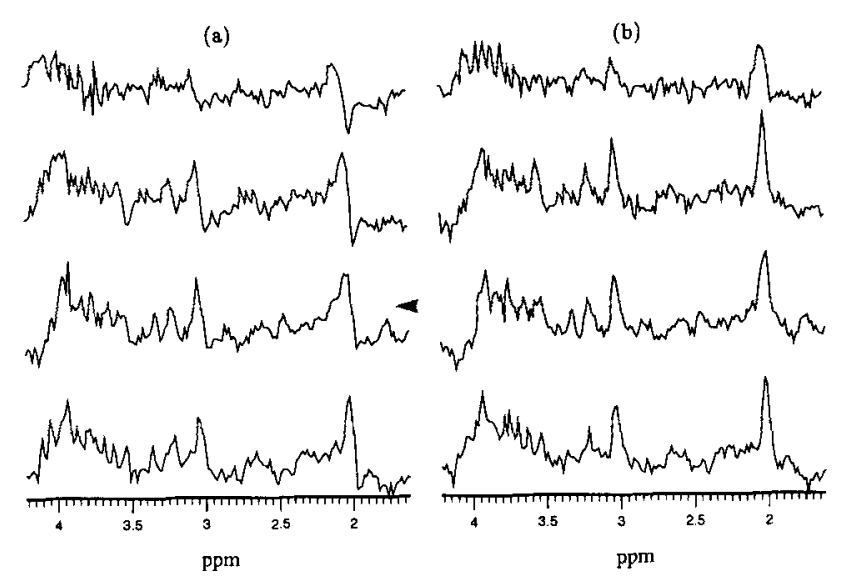

FIG. 8. In vivo correction using fewer phase cycles reference CSI. Data were acquired using a $6 \times 2 \times 2 \mathrm{~cm}$ STEAM voxel localized in the occipital cortex with a 2000-ms $T R$, a 20-ms $T E$, and a $11 \mathrm{~ms}$ TM. Sample CSI data were acquired in 8:32 using $16 \mathrm{PEs}$ and 8 phase cycles/PE. Phase encoding produced $41.5 \times 2 \times 2 \mathrm{~cm}$ subvoxels. (a) Manually phased (uncorrected) spectra. The arrow indicates the spectrum selected for manual phasing. A zero-order phase correction of $340^{\circ}$ was applied to each spectrum. (b) Corrected spectra. Reference CSI data were acquired in 1:20 using a 2000-ms TR, 16 PEs and 2 phase cycles/PE. Spectra are referenced to NAA at $2.02 \mathrm{ppm}$. 
of performing a reference correction on a sample CSI taken from the occipital lobe in human brain. To minimize the possibility of inadequate correction, an identical number of PEs were used in the reference. Note that the asymmetric line shape of the $N$-acetyl aspartate (NAA) peak has been corrected in each signal. Also note that the NAA, creatine/phosphocreatine ( $\mathrm{Cr} / \mathrm{PCr}$ ), and choline (Cho) resonances are each referenced to a common resonance frequency. The approximation should improve as voxel size decreases and the importance of the spatial dependence of the eddy current contribution decreases. Adequate SNR is required, however, in each reference signal to obtain an accurate estimate of the aggregate reference phase. This is especially important at early time points as the SNR of the corrected sample CSI can be adversely affected.

We have shown that certain simplifying approximations can yield reasonable phase correction with reductions in reference data acquisition times of up to a factor of eight. Though not shown here, restoring missing reference PEs (from 8 to 16 ) resulted in better performance of the phase correction algorithm at the expense of additional acquisition time. This suggests that additional time savings may be obtained from spatial approximations of the reference CSI. This may be unnecessary with 1D-CSI, where the identical reference required for optimal phase correction can be acquired without substantial time penalty. Multidimensional CSI, however, would benefit significantly from such spatial approximations. In this preliminary work, we have utilized 1D-CSI and a linear sampling/interpolation of $k$-space for the reference CSI. Future work will focus on using multidimensional CSI in conjunction with nonlinear sampling/interpolation of $k$ space where a reduced number of PEs cover a larger range of spatial frequencies. This may provide an improvement in eddy current correction per unit reference CSI acquisition time, particularly in vivo where the magnetic field inhomogeneity and eddy current distribution is more pronounced.

\section{ACKNOWLEDGMENTS}

The authors thank David Gurr, Ralph Hurd, Sue Kohler, Napapon Sailasuta, and Peter Webb of GE Medical Systems for their assistance, and the reviewers for their thoughtful comments and suggestions.

\section{REFERENCES}

1. J. W. Hugg, J. H, Duijn, G. B. Matson, A. A. Maudsley, J. S. Tsuruda, D. F. Gelinas, M. W. Weiner, J. Cereb. Blood Flow Metab. 12, 734 (1992).

2. J. H. Duijn, G. B. Matson, A. A. Maudsley, J. W. Hugg, M. W. Weiner, Radiology 183, 711 (1992).

3. J. H. Duijn, G. B. Matson, A. A. Maudsley, M. W. Weiner, Magn. Reson. Imaging 10, 315 (1992).

4. D. M. Spielman, J. M. Pauly, A. Macovski, G. H. Glover, D. R. Enzmann, J. Magn. Reson. Imaging 2, 253 (1992).

5. G. D. Graham, A. M. Blamire, A. M. Howseman, D. L. Rothman, P. B. Fayad, L. M. Brass, O. A. Petroff, R. G. Shulman, J. W. Pritchard, Stroke 23, 333 (1992).

6. C. C. Ford, R. H. Griffey, N. A. Matwiyoff, G. A. Rosenberg, Neurology 42, 1408 (1992).

7. P. R. Luyten, A. J. H. Marien, W. Heindel, P. H. J. van Gerwen, K. Herholz, J. A. den Hollander, G. Freidmann, W. D. Heiss, Radiology 176, 791 (1990).

8. C. M. Segebarth, D. F. Baleriaux, P. R. Luyten, J. A. den Hollander, Magn. Reson. Med. 13, 62 (1990).

9. A. A. Maudsley, S. K. Hilal, W. H. Perman, H. E. Símon, J. Magn. Reson. 51, 147 (1983).

10. T. R. Brown, B. M. Kincaid, K. Uğurbil, Proc. Natl. Acad. Sci. (U.S.A.) 79, 3523 (1982).

11. P. Webb, D. Spielman, A. Macovski, Magn. Reson. Med. 23, 1 (1992).

12. D. Spielman, P. Webb, A. Macovski, Magn. Reson. Med. 12, 38 (1989).

13. A. A. Maudsley, S. K. Hilal, Magn. Reson. Med. 2, 218 (1985).

14. R. J. Ordidge, I. D. Cresshull, J. Magn. Reson. 69, 151 (1986).

15. P. Jehenson, A. Syrota, Magn. Reson. Med. 12, 253 (1989).

16. U. Klose, Magn. Reson. Med. 14, 26 (1990).

17. W. R. Riddle, S. J. Gibbs, M. R. Willcott, Med. Phys. 19, 501 (1992).

18. J. Frahm, K. D. Merboldt, W. Hanicke, J. Magn. Reson. 72, 502 (1987).

19. E. Schneider, G. Glover, Magn. Reson. Med. 18, 335 (1991).

20. A. Haase, J. Frahm, W. Hanicke, D. Matthaei, Phys. Med. Biol. 30, 341 (1985).

21. J. Pauly, P. LeRoux, D. Nishimura, A. Macovski, IEEE Trans. Med. Imaging 10, 53 (1991). 